\title{
Limiting Behavior of the Maximum of the Partial Sum for Linearly Negative Quadrant Dependent Random Variables under Residual Cesàro Alpha-Integrability Assumption
}

\author{
Jiangfeng Wang and Qunying Wu \\ College of Science, Guilin University of Technology, Guilin 541004, China \\ Correspondence should be addressed to Qunying Wu, wqy666@glite.edu.cn
}

Received 13 September 2011; Accepted 29 December 2011

Academic Editor: Xianhua Tang

Copyright (C) 2012 J. Wang and Q. Wu. This is an open access article distributed under the Creative Commons Attribution License, which permits unrestricted use, distribution, and reproduction in any medium, provided the original work is properly cited.

Linearly negative quadrant dependence is a special dependence structure. By relating such conditions to residual Cesàro alpha-integrability assumption, as well as to strongly residual Cesàro alpha-integrability assumption, some $L_{p}$-convergence and complete convergence results of the maximum of the partial sum are derived, respectively.

\section{Introduction}

The classical notion of uniform integrability of a sequence $\left\{X_{n}\right\}_{n \in \mathbb{N}}$ of integrable random variables is defined through the condition $\lim _{a \rightarrow \infty} \sup _{n \geq 1} E\left|X_{n}\right| I\left(\left|X_{n}\right|>a\right)=0$. Landers and Rogge [1] proved that the uniform integrability condition is sufficient in order that a sequence of pairwise independent random variables verifies the weak law of large numbers (WLLNs). Chandra [2] weakened the assumption of uniform integrability to Cesáro uniform integrability (CUI) and obtained $L_{1}$-convergence for pairwise independent random variables.

Chandra and Goswami [3] improved the above-mentioned result of Landers and Rogge [1]. They showed that for a sequence of pairwise independent random variables, CUI is sufficient for the WLLN to hold and strong Cesáro uniform integrability (SCUI) is sufficient for the strong law of large numbers (SLLNs) to hold. Landers and Rogge [4] obtained a slight improvement over the results of Chandra [2] and Chandra and Goswami [3] for the case of nonnegative random variables. They showed that, in this case, the condition of pairwise independence can be replaced by the weaker assumption of pairwise nonpositive correlation.

Chandra and Goswami [5] introduced a new set of conditions called Cesáro $\alpha$-integrability $(\mathrm{CI}(\alpha))$ and strong Cesáro $\alpha$-integrability $(\mathrm{SCI}(\alpha))$ for a sequence of random 
variables, which are strictly weaker than CUI and SCUI, respectively. They showed that, for $\alpha<1 / 2, \mathrm{CI}(\alpha)$ is sufficient for the WLLN to hold and SCI $(\alpha)$ is sufficient for the SLLN to hold for a sequence of pairwise independent random variables, which are improvements over the results of Landers and Rogge [4] and the earlier results.

Chandra and Goswami [6] relaxed the condition of $\mathrm{CI}(\alpha)$ to residual Cesáro alphaintegrability ( $\mathrm{RCI}(\alpha)$, see Definition 2.1 below) and the condition of SCI $(\alpha)$ to strong residual Cesáro alpha-integrability (SRCI $(\alpha)$, see Definition 2.3 below) and significantly improved the results of Chandra and Goswami [5].

Recently, Yuan and $\mathrm{Wu}$ [7] discussed some limiting behaviors of the maximum of partial sum for asymptotically negatively associated random variables when such random variables are subject to $\operatorname{RCI}(\alpha)$ and $\operatorname{SRCI}(\alpha)$.

In this paper, we will derive some $L_{p}$-convergence and complete convergence of the maximum of partial sum for linearly negative quadrant dependent random variables when such random variables are subject to $\mathrm{RCI}(\alpha)$ and $\mathrm{SRCI}(\alpha)$. These results generalize previous work in the literature.

\section{Preliminaries}

First let us specify the two special kinds of uniform integrability we are dealing with in the subsequent sections, which were introduced by Chandra and Goswami [6].

Definition 2.1. For $\alpha \in(0, \infty)$, a sequence $\left\{X_{n}\right\}_{n \in \mathbb{N}}$ of random variables is said to be residual Cesáro alpha-integrable $(\operatorname{RCI}(\alpha)$, in short) if

$$
\sup _{n \geq 1} \frac{1}{n} \sum_{i=1}^{n} E\left|X_{i}\right|<\infty, \quad \lim _{n \rightarrow \infty} \frac{1}{n} \sum_{i=1}^{n} E\left(\left|X_{i}\right|-i^{\alpha}\right) I\left(\left|X_{i}\right|>i^{\alpha}\right)=0
$$

Clearly, $\left\{X_{n}\right\}$ is $\operatorname{RCI}(\alpha)$ for any $\alpha>0$ if $\left\{X_{n}\right\}_{n \in \mathbb{N}}$ is identically distributed with $E\left|X_{1}\right|<$ $\infty$, and $\left\{\left|X_{n}\right|^{p}\right\}_{n \in \mathbb{N}}$ is $\operatorname{RCI}(\alpha)$ for any $\alpha>0$ if $\left\{X_{n}\right\}_{n \in \mathbb{N}}$ is stochastically dominated by a nonnegative random variable $X$ with $E X^{p}<\infty$ for some $p \geq 1$.

Definition 2.2. For $\alpha \in(0, \infty)$, a sequence $\left\{X_{n}\right\}_{n \in \mathbb{N}}$ of random variables is said to be strongly residual Cesáro alpha-integrable $(\operatorname{SRCI}(\alpha)$, in short) if

$$
\sup _{n \geq 1} \frac{1}{n} \sum_{i=1}^{n} E\left|X_{i}\right|<\infty, \quad \sum_{n=1}^{\infty} \frac{1}{n} E\left(\left|X_{n}\right|-n^{\alpha}\right) I\left(\left|X_{n}\right|>n^{\alpha}\right)<\infty .
$$

We point out that, $\left\{\left|X_{n}\right|^{p}\right\}_{n \in \mathbb{N}}$ is $\operatorname{SRCI}(\alpha)$ for any $\alpha>0$, provided that $\left\{X_{n}\right\}_{n \in \mathbb{N}}$ is stochastically dominated by a nonnegative random variable $X$ with $E X^{p+\delta}<\infty$ for some $p \geq 1$ and $\delta>0$.

The condition of $\operatorname{SRCI}(\alpha)$ is a "strong" version of the condition of $\operatorname{RCI}(\alpha)$. Moreover, for any $\alpha>0, \operatorname{RCI}(\alpha)$ is strictly weaker than $\mathrm{CI}(\alpha)$, thereby weaker than $\mathrm{CUI}$, while $\operatorname{SRCI}(\alpha)$ is strictly weaker than $\mathrm{SCI}(\alpha)$, thereby much weaker than SCUI.

Next, we turn our attention to the dependence structure for random variables. For our purpose, we have to mention a special kind of dependence, namely, negative quadrant dependence. 
Definition 2.3 (cf. Lehmann [8]). Two random variables $X$ and $Y$ are said to be negative quadrant dependent (NQD, in short) if for any $x, y \in \mathbb{R}$,

$$
P(X<x, Y<y) \leq P(X<x) P(Y<y) .
$$

A sequence $\left\{X_{n}\right\}_{n \in \mathbb{N}}$ of random variables is said to be pairwise NQD if $X_{i}$ and $X_{j}$ are NQD for all $i, j \in \mathbb{N}^{+}$and $i \neq j$.

Definition 2.4 (cf. Newman [9]). A sequence $\left\{X_{n}\right\}_{n \in \mathbb{N}}$ of random variables is said to be linearly negative quadrant dependent (LNQD, in short) if for any disjoint subsets $A, B \in \mathbb{Z}^{+}$and positive $r_{j}^{\prime} s$,

$$
\sum_{k \in A} r_{k} X_{k}, \quad \sum_{j \in B} r_{j} X_{j} \text { are NQD. }
$$

Remark 2.5. It is easily seen that if $\left\{X_{n}\right\}_{n \in \mathbb{N}}$ is a sequence of LNQD random variables, then $\left\{a X_{n}+b\right\}_{n \in \mathbb{N}}$ is still a sequence of LNQD random variables, where $a$ and $b$ are real numbers.

The concept of LNQD sequence was introduced by Newman [9]. Some applications for LNQD sequence have been found; see, for example, the work by Newman [9] who established the central limit theorem for a strictly stationary LNQD process. Wang and Zhang [10] provided uniform rates of convergence in the central limit theorem for LNQD sequence. Ko et al. [11] obtained the Hoeffding-type inequality for LNQD sequence. Ko et al. [12] studied the strong convergence for weighted sums of LNQD arrays. Fu and $\mathrm{Wu}$ [13] studied the almost sure central limit theorem for LNQD sequences, and so forth. We note that "«" means "O."

Lemma 2.6 (cf. Lehmann [8]). Let random variables $X$ and $Y$ be NQD. Then

(1) EXY $\leq E X E Y$;

(2) $P(X<x, Y<y) \leq P(X<x) P(Y<y)$;

(3) If $f$ and $g$ are both nondecreasing (or both nonincreasing) functions, then $f(X)$ and $g(Y)$ are $N Q D$.

Lemma 2.7 (cf. Hu et al. [14]). Let $\left\{X_{n}\right\}_{n \in \mathbb{N}}$ be a LNQD sequence of random variables with $E X_{n}=$ 0 . Assume that there exists a $p>2$ satisfying $E\left|X_{i}\right|^{p}<\infty$ for every $i \geq 1$. Then, there exists a positive constant $c$ such that

$$
E\left(\max _{1 \leq k \leq n}\left|\sum_{i=1}^{k} X_{i}\right|\right)^{p} \leq c A(p)\left(\sum_{i=1}^{n}\left(E\left|X_{i}\right|^{p}\right)^{2 / p}\right)^{p / 2}, \quad \forall n \geq 1,
$$

where $A(p)=A_{p, p / 2}$ is a positive constant depending only on $p$.

It is easily seen that when $p=2$, the above equation still holds true. 
Lemma 2.8. Let $\left\{X_{n}\right\}_{n \in \mathbb{N}}$ be LNQD random variables sequences with mean zero. Then for $1<p<2$, there exists a positive constant $c$ such that

$$
E\left(\max _{1 \leq i \leq n}\left|S_{i}\right|^{p}\right) \leq c\left(\sum_{i=1}^{n}\left(E\left|X_{i}\right|^{p}\right)^{1 / p}\right)^{p}, \quad \forall n \geq 1 .
$$

This lemma is easily proved by the results of Zhang [15] and Yuan and Wu [7]. Here we omit the details of the proof.

Lemma 2.9. Let $\left\{X_{k}\right\}_{k \in \mathbb{N}^{d}}$ be a centered $L N Q D$ random field. Then for any $p>1$, there exists a positive constant $c$ such that

$$
E\left|\sum_{i=1}^{n} X_{i}\right|^{p} \leq c \sum_{i=1}^{n} E\left|X_{i}\right|^{p}
$$

for all $n \geq 1$.

This lemma is due to Zhang [15, Lemma 3.3].

Finally, we give a lemma which supplies us with the analytical part in the proofs of theorems in the subsequent sections.

Lemma 2.10 (cf. Landers and Rogge [4]). For sequences $\left\{a_{n}\right\}_{n \in \mathbb{N}}$ and $\left\{b_{n}\right\}_{n \in \mathbb{N}}$ of nonnegative real numbers, if

$$
\sup _{n \geq 1} n^{-1} \sum_{i=1}^{n} a_{i}<\infty, \quad \sum_{n=1}^{\infty} b_{n}<\infty
$$

then

$$
\sum_{i=1}^{n} a_{i} b_{i} \leq\left(\sup _{m \geq 1} m^{-1} \sum_{i=1}^{m} a_{i}\right) \sum_{i=1}^{n} b_{i}<\infty,
$$

for every $n \geq 1$.

\section{Residual Cesáro Alpha-Integrability and $L_{p}$-Convergence of the Maximum of the Partial Sum}

Let $p>1$, and let $h(x)$ be a strictly positive function defined on $(1,+\infty)$. In this section, we discuss $L_{p}$-convergence of the form of $n^{-h(p)} \max _{1 \leq i \leq n}\left|S_{i}-E S_{i}\right|$ for a LNQD sequence $\left\{X_{n}\right\}_{n \in \mathbb{N}}$ of random variables, provided that $\left\{\left|X_{n}\right|^{p}\right\}_{n \in \mathbb{N}}$ is $\operatorname{RCI}(\alpha)$ for an appropriate condition.

Our first result is dealing with the case $1<p<2$.

Theorem 3.1. Let $1<p<2$, and let $\left\{X_{n}\right\}_{n \in \mathbb{N}}$ be a LNQD sequence of random variables. If $\left\{\left|X_{n}\right|^{p}\right\}_{n \in \mathbb{N}}$ is $\operatorname{RCI}(\alpha)$ for some $\alpha \in(0,1 /(2-p))$, then

$$
n^{-1} \max _{1 \leq i \leq n}\left|S_{i}-E S_{i}\right| \longrightarrow 0 \quad \text { in } L_{p}
$$


Proof of Theorem 3.1. Let $Y_{n}=-n^{\alpha} I\left(X_{n}<-n^{\alpha}\right)+X_{n} I\left(\left|X_{n i}\right| \leq n^{\alpha}\right)+n^{\alpha} I\left(X_{n}>n^{\alpha}\right), n \geq 1$, and define, for each $n \geq 1, Z_{n}=X_{n}-Y_{n}, S_{n}^{(1)}=\sum_{i=1}^{n} Y_{i}$, and $S_{n}^{(2)}=\sum_{i=1}^{n} Z_{i}$. It is easy to see that $\left|Y_{n}\right|=\min \left\{\left|X_{n}\right|, n^{\alpha}\right\},\left|Z_{n}\right|=\left(\left|X_{n}\right|-n^{\alpha}\right) I\left(\left|X_{n}\right|>n^{\alpha}\right)$, and

$$
\left|Z_{n}\right|^{p} \leq\left(\left|X_{n}\right|^{p}-n^{\alpha}\right) I\left(\left|X_{n}\right|^{p}>n^{\alpha}\right)
$$

for all $p>1$. Note that, for each $n \geq 1, Y_{n}$ and $Z_{n}$ are monotone transformations of the initial variable $X_{n}$. This implies that LNQD assumption is preserved by this construction in view of Lemma 2.6. Precisely, $\left\{Y_{n}-E Y_{n}\right\}_{n \in \mathbb{N}}$ and $\left\{Z_{n}-E Z_{n}\right\}_{n \in \mathbb{N}}$ are also LNQD sequences of zero mean random variables.

For our purpose, it suffices to prove

$$
\begin{aligned}
& n^{-1} \max _{1 \leq i \leq n}\left|S_{i}^{(1)}-E S_{i}^{(1)}\right| \longrightarrow 0 \quad \text { in } L_{2} \\
& n^{-1} \max _{1 \leq i \leq n}\left|S_{i}^{(2)}-E S_{i}^{(2)}\right| \longrightarrow 0 \quad \text { in } L_{p} .
\end{aligned}
$$

Using Lemma 2.8, the Hölder inequality, relation (3.2), and the second condition in (2.1) of the $\operatorname{RCI}(\alpha)$ property of the sequence $\left\{\left|X_{n}\right|^{p}\right\}_{n \in \mathbb{N}}$, we obtain

$$
\begin{aligned}
n^{-p} E\left(\max _{1 \leq i \leq n}\left|S_{i}^{(2)}-E S_{i}^{(2)}\right|^{p}\right) & \ll n^{-p}\left(\sum_{i=1}^{n}\left(E\left|Z_{i}-E Z_{i}\right|^{p}\right)^{1 / p}\right)^{p} \\
& \ll n^{-1} \sum_{i=1}^{n} E\left|Z_{i}-E Z_{i}\right|^{p} \ll n^{-1} \sum_{i=1}^{n} E\left|Z_{i}\right|^{p} \\
& \leq n^{-1} \sum_{i=1}^{n} E\left(\left|X_{i}\right|^{p}-i^{\alpha}\right) I\left(\left|X_{i}\right|^{p}>i^{\alpha}\right) \longrightarrow 0 .
\end{aligned}
$$

This proves (3.4). To verify (3.3), using Lemma 2.7, we have

$$
\begin{aligned}
n^{-2} E\left(\max _{1 \leq i \leq n}\left|S_{i}^{(1)}-E S_{i}^{(1)}\right|^{2}\right) & \ll n^{-2}\left(\sum_{i=1}^{n} E\left(Y_{i}-E Y_{i}\right)^{2}\right) \\
& \ll n^{-2} \sum_{i=1}^{n} E Y_{i}^{2} \leq n^{-2+(2-p) \alpha} \sum_{i=1}^{n} E\left|X_{i}\right|^{p} \\
& \leq n^{-1+(2-p) \alpha} \cdot \sup _{n \geq 1}\left(n^{-1} \sum_{i=1}^{n} E\left|X_{i}\right|^{p}\right) .
\end{aligned}
$$

Using the first condition of (2.1) of the $\operatorname{RCI}(\alpha)$ property of the sequence $\left\{\left|X_{n}\right|^{p}\right\}_{n \in \mathbb{N}}$, the last expression above clearly goes to 0 as $n \rightarrow \infty$, from $1<p<2$ and $\alpha<1 /(2-p)$, thus completing the proof.

Remark 3.2. Let $1<p<2$, and let $\left\{X_{n}\right\}_{n \in \mathbb{N}}$ be a LNQD sequence of random variables. If $\left\{\left|X_{n}\right|^{p}\right\}_{n \in \mathbb{N}}$ is $\operatorname{RCI}(\alpha)$ for some $\alpha \in(0,1 / p)$, then $n^{-1 / p}\left(S_{n}-E S_{n}\right) \rightarrow 0$ in $L_{p}$. 
Compared with Theorem 3.1, this result, whose proof can be completed by using Lemma 2.9, drops the maximum of the partial sum at the price of enlarging $1 / n$ into $1 / n^{1 / p}$. Next we consider the case $p \geq 2$.

Theorem 3.3. Let $p \geq 2$, and let $\left\{X_{n}\right\}_{n \in \mathbb{N}}$ be a LNQD sequence of random variables. If $\left\{X_{n}\right\}_{n \in \mathbb{N}}$ satisfies

$$
\sup _{n \geq 1} \frac{1}{n} \sum_{i=1}^{n} E\left|X_{i}\right|^{p}<\infty
$$

then for any $\delta>1 / 2$

$$
n^{-\delta} \max _{1 \leq i \leq n}\left|S_{i}-E S_{i}\right| \longrightarrow 0 \quad \text { in } L_{p}
$$

Proof of Theorem 3.3. By Lemma 2.7 and the Hölder inequality,

$$
\begin{aligned}
E\left(n^{-\delta} \max _{1 \leq i \leq n}\left|S_{i}-E S_{i}\right|\right)^{p} & \ll n^{-p \delta}\left(\sum_{i=1}^{n}\left(E\left|X_{i}\right|^{p}\right)^{2 / p}\right)^{p / 2} \\
& \leq n^{-p \delta+(p / 2)-1} \sum_{i=1}^{n} E\left|X_{i}\right|^{p} \\
& \leq n^{-p \delta+(p / 2)} \cdot \sup _{n \geq 1} \frac{1}{n} \sum_{i=1}^{n} E\left|X_{i}\right|^{p} \longrightarrow 0 .
\end{aligned}
$$

The proof is completed.

\section{Strongly Residual Cesáro Alpha-Integrability and Complete Convergence of the Maximum of the Partial Sum}

A sequence of random variables $\left\{X_{n}\right\}_{n \in \mathbb{N}}$ is said to converge completely to a constant $a$ if for any $\varepsilon>0$,

$$
\sum_{n=1}^{\infty} P\left(\left|X_{n}-a\right|>\varepsilon\right)<\infty
$$

In this case we write $X_{n} \rightarrow a$ completely. This notion was given by Hsu and Robbins [16]. Note that the complete convergence implies the almost sure convergence in view of the BorelCantelli lemma.

The condition of $\operatorname{SRCI}(\alpha)$ is a strong version of the condition of $\operatorname{RCI}(\alpha)$. In this section, we will show that each of the theorems in the previous section has a corresponding "strong" analogue in the sense of complete convergence.

Theorem 4.1. Let $1<p<2$, and let $\left\{X_{n}\right\}_{n \in \mathbb{N}}$ be a $L N Q D$ sequence of random variables. If $\left\{\left|X_{n}\right|^{p}\right\}_{n \in \mathbb{N}}$ is $\operatorname{SRCI}(\alpha)$ for some $\alpha \in(0,1 /(2-p))$, then

$$
n^{-1} \max _{1 \leq i \leq n}\left|S_{i}-E S_{i}\right| \longrightarrow 0 \text { completely } .
$$


Proof of Theorem 4.1. For any $n \geq 1$, let $m=m_{n}$ be the integer such that $2^{m-1}<n \leq 2^{m}$. Observe that

$$
\begin{aligned}
n^{-1} \max _{1 \leq i \leq n}\left|S_{i}-E S_{i}\right| & \leq n^{-1} \max _{1 \leq i \leq 2^{m}}\left|S_{i}-E S_{i}\right| \\
& \leq\left(2^{m-1}\right)^{-1} \max _{1 \leq i \leq 2^{m}}\left|S_{i}-E S_{i}\right| \\
& =2 \cdot 2^{-m} \max _{1 \leq i \leq 2^{m}}\left|S_{i}-E S_{i}\right| .
\end{aligned}
$$

Hence it suffices to show that

$$
2^{-m} \max _{1 \leq i \leq 2^{m}}\left|S_{i}-E S_{i}\right| \longrightarrow 0 \text { completely. }
$$

Let $Y_{n}, Z_{n}, S_{n}^{(1)}$, and $S_{n}^{(2)}$ be defined as in the proof of Theorem 3.1. We first prove that $2^{-m} \max _{1 \leq i \leq 2^{m}}\left|S_{i}^{(2)}-E S_{i}^{(2)}\right| \rightarrow 0$ completely; that is,

$$
2^{-m} \max _{1 \leq i \leq 2^{m}}\left|\sum_{k=1}^{i}\left(Z_{k}-E Z_{k}\right)\right| \longrightarrow 0 \text { completely. }
$$

Using Lemma 2.8, the Hölder inequality, relation (3.2), and the second condition in (2.1) of the $\operatorname{RCI}(\alpha)$ property of the sequence $\left\{\left|X_{n}\right|^{p}\right\}_{n \in \mathbb{N}}$, we have

$$
\begin{aligned}
\sum_{m=0}^{\infty} E\left(2^{-m} \max _{1 \leq i \leq 2^{m}}\left|\sum_{k=1}^{i}\left(Z_{k}-E Z_{k}\right)\right|\right)^{p} & \ll \sum_{m=0}^{\infty} 2^{-m p}\left(\sum_{i=1}^{2^{m}}\left(E\left|Z_{i}\right|^{p}\right)^{1 / p}\right)^{p} \\
& \leq \sum_{m=0}^{\infty} 2^{-m} \sum_{i=1}^{2^{m}} E\left|Z_{i}\right|^{p} \\
& =\sum_{i=1}^{\infty} E\left|Z_{i}\right|^{p} \sum_{m: 2^{m} \geq i} 2^{-m} \\
& \leq \sum_{i=1}^{\infty} i^{-1} E\left|Z_{i}\right|^{p} \\
& \leq \sum_{i=1}^{\infty} i^{-1} E\left(\left|X_{i}\right|^{p}-i^{\alpha}\right) I\left(\left|X_{i}\right|^{p}>i^{\alpha}\right) \\
& <\infty,
\end{aligned}
$$

which implies (4.4).

Next we show that $2^{-m} \max _{1 \leq i \leq 2^{m}}\left|S_{i}^{(1)}-E S_{i}^{(1)}\right| \rightarrow 0$ completely; that is,

$$
2^{-m} \max _{1 \leq i \leq 2^{m}}\left|\sum_{k=1}^{i}\left(Y_{k}-E Y_{k}\right)\right| \longrightarrow 0 \text { completely. }
$$


By Lemma 2.7 and the Hölder inequality,

$$
\begin{aligned}
\sum_{m=0}^{\infty} E\left(2^{-m} \max _{1 \leq i \leq 2^{m}} \sum_{k=1}^{i}\left(Y_{k}-E Y_{k}\right)\right)^{2} & \ll \sum_{m=0}^{\infty} 2^{-2 m} \sum_{i=1}^{2^{m}} E Y_{i}^{2} \\
& \leq \sum_{m=0}^{\infty} 2^{-2 m} \sum_{i=1}^{2^{m}} i^{(2-p) \alpha} E\left|X_{i}\right|^{p}
\end{aligned}
$$

In view of the first condition in (2.1) of the $\operatorname{RCI}(\alpha)$ property of the sequence $\left\{\left|X_{n}\right|^{p}\right\}_{n \in \mathbb{N}}$, we have

$$
\begin{aligned}
\sum_{m=0}^{\infty} E\left(2^{-m} \max _{1 \leq i \leq 2^{m}} \sum_{k=1}^{i}\left(Y_{k}-E Y_{k}\right)\right)^{2} & \leq \sum_{m=0}^{\infty} 2^{-2 m} \sum_{i=1}^{2^{m}} i^{(2-p) \alpha} E\left|X_{i}\right|^{p} \\
& \leq \sum_{m=0}^{\infty} 2^{-2 m} \cdot 2^{m(2-p) \alpha} \sum_{i=1}^{2^{m}} E\left|X_{i}\right|^{p} \\
& \leq \sum_{m=0}^{\infty} 2^{-m} \cdot 2^{m(2-p) \alpha} \cdot \sup _{m: 2^{m} \geq i} \frac{1}{2^{m}} \sum_{i=1}^{2^{m}} E\left|X_{i}\right|^{p} .
\end{aligned}
$$

The last series above converges since $\alpha \in(0,1 /(2-p))$ implies $-1+(2-p) \alpha<0$, and therefore (4.7) holds. This completes the proof.

For the case $p \geq 2$, we have the following result.

Theorem 4.2. Let $p \geq 2$, and let $\left\{X_{n}\right\}_{n \in \mathbb{N}}$ be a LNQD sequence of random variables. If $\left\{X_{n}\right\}_{n \in \mathbb{N}}$ satisfies

$$
\sup _{n \geq 1} \frac{1}{n} \sum_{i=1}^{n} E\left|X_{i}\right|^{p}<\infty
$$

then for any $\delta>1 / 2$

$$
n^{-\delta} \max _{1 \leq i \leq n}\left|S_{i}-E S_{i}\right| \longrightarrow 0 \text { completely }
$$

Proof of Theorem 4.2. Let $m_{n}, n \geq 1$ be defined as in the proof of Theorem 4.1. Proceeding in the proof of (4.3), we see that it suffices to show that

$$
2^{-m \delta} \max _{1 \leq i \leq 2^{m}}\left|S_{i}-E S_{i}\right| \longrightarrow 0 \text { completely. }
$$


Indeed by Lemma 2.7 and the Hölder inequality,

$$
\begin{aligned}
\sum_{m=0}^{\infty} E\left(2^{-m \delta} \max _{1 \leq i \leq 2^{m}}\left|S_{i}-E S_{i}\right|\right)^{p} & \ll \sum_{m=0}^{\infty} 2^{-m p \delta}\left(\sum_{i=1}^{2^{m}}\left(E\left|X_{i}\right|^{p}\right)^{2 / p}\right)^{p / 2} \\
& \leq \sum_{i=1}^{\infty} E\left|X_{i}\right|^{p} \sum_{m: 2^{m} \geq i} 2^{-m p \delta-m+2 m / p} \\
& \leq \sum_{i=1}^{\infty} i^{-p \delta-1+p / 2} E\left|X_{i}\right|^{p}
\end{aligned}
$$

In view of Lemma 2.10,

$$
\begin{aligned}
\sum_{i=1}^{\infty} i^{-p \delta-1+p / 2} E\left|X_{i}\right|^{p} & \leq \sup _{n \geq 1} \frac{1}{n} \sum_{i=1}^{2^{m}} E\left|X_{i}\right|^{p} \sum_{n=1}^{\infty} n^{-p \delta-1+p / 2} \\
& \ll \sum_{n=1}^{\infty} n^{-p \delta-1+p / 2}<\infty
\end{aligned}
$$

from $-p \delta-1+p / 2<-1$. Therefore (4.12) holds. The proof is completed.

\section{Acknowledgments}

Supported by the National Science Foundation of China (11061012), the Guangxi China Science Foundation (2010GXNSFA013120), and Innovation Project of Guangxi Graduate Education (2010105960202M32). We are very grateful to the referees and the editors for their valuable comments and some helpful suggestions that improved the clarity and readability of the paper.

\section{References}

[1] D. Landers and L. Rogge, "Laws of large numbers for pairwise independent uniformly integrable random variables," Mathematische Nachrichten, vol. 130, pp. 189-192, 1987.

[2] T. K. Chandra, "Uniform integrability in the Cesàro sense and the weak law of large numbers," Sankhyā. Series A, vol. 51, no. 3, pp. 309-317, 1989.

[3] T. K. Chandra and A. Goswami, "Cesàro uniform integrability and the strong law of large numbers," Sankhyā. Series A, vol. 54, no. 2, pp. 215-231, 1992.

[4] D. Landers and L. Rogge, "Laws of large numbers for uncorrelated Cesàro uniformly integrable random variables," Sankhyā. Series A, vol. 59, pp. 301-310, 1997.

[5] T. K. Chandra and A. Goswami, "Cesàro $\alpha$-integrability and laws of large numbers. I," Journal of Theoretical Probability, vol. 16, no. 3, pp. 655-669, 2003.

[6] T. K. Chandra and A. Goswami, "Cesàro $\alpha$-integrability and laws of large numbers. II," Journal of Theoretical Probability, vol. 19, no. 4, pp. 789-816, 2006.

[7] D. M. Yuan and X. S. Wu, "Limiting behavior of the maximum of the partial sum for asymptotically negatively associated random variables under residual Cesàro alpha-integrability assumption," Journal of Statistical Planning and Inference, vol. 140, no. 9, pp. 2395-2402, 2010.

[8] E. L. Lehmann, "Some concepts of dependence," Annals of Mathematical Statistics, vol. 37, pp. 11371153, 1966. 
[9] C. M. Newman, "Asymptotic independence and limit theorems for positively and negatively dependent random variables," in Inequalities in Statistics and Probability, Y. L. Tong, Ed., vol. 5 of IMS Lecture Notes Monogr. Ser., pp. 127-140, Inst. Math. Statist., Hayward, Calif, USA, 1984.

[10] J. F. Wang and L. X. Zhang, "A Berry-Esseen theorem for weakly negatively dependent random variables and its applications," Acta Mathematica Hungarica, vol. 110, no. 4, pp. 293-308, 2006.

[11] M.-H. Ko, Y.-K. Choi, and Y.-S. Choi, "Exponential probability inequality for linearly negative quadrant dependent random variables," Korean Mathematical Society. Communications, vol. 22, no. 1, pp. 137-143, 2007.

[12] M.-H. Ko, D.-H. Ryu, and T.-S. Kim, "Limiting behaviors of weighted sums for linearly negative quadrant dependent random variables," Taiwanese Journal of Mathematics, vol. 11, no. 2, pp. 511-522, 2007.

[13] Y. Fu and Q. Wu, "Almost sure central limit theorem for LNQD sequences," Journal of Guilin University of Technology, vol. 30, no. 4, pp. 637-639, 2010.

[14] S. Hu, X. Li, W. Yang, and X. Wang, "Maximal inequalities for some dependent sequences and their applications," Journal of the Korean Statistical Society, vol. 40, no. 1, pp. 11-19, 2011.

[15] L.-X. Zhang, "A functional central limit theorem for asymptotically negatively dependent random fields," Acta Mathematica Hungarica, vol. 86, no. 3, pp. 237-259, 2000.

[16] P. L. Hsu and H. Robbins, "Complete convergence and the law of large numbers," Proceedings of the National Academy of Sciences of the United States of America, vol. 33, pp. 25-31, 1947. 


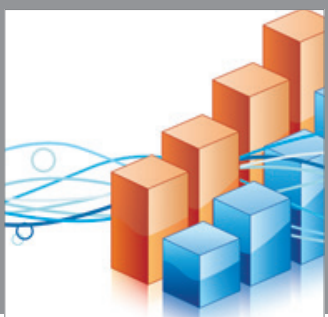

Advances in

Operations Research

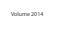

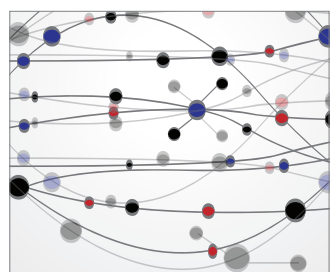

\section{The Scientific} World Journal
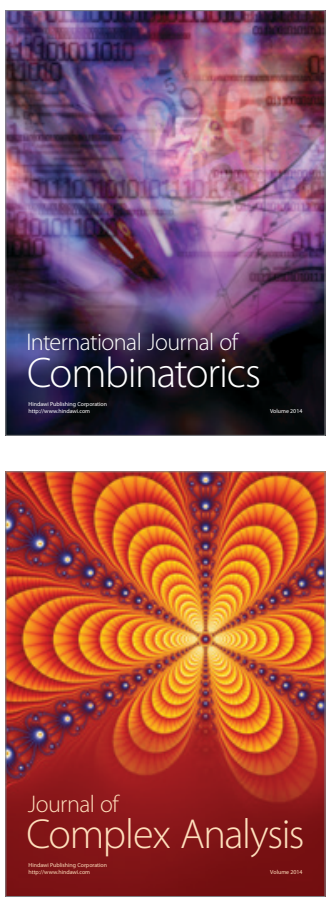

International Journal of

Mathematics and

Mathematical

Sciences
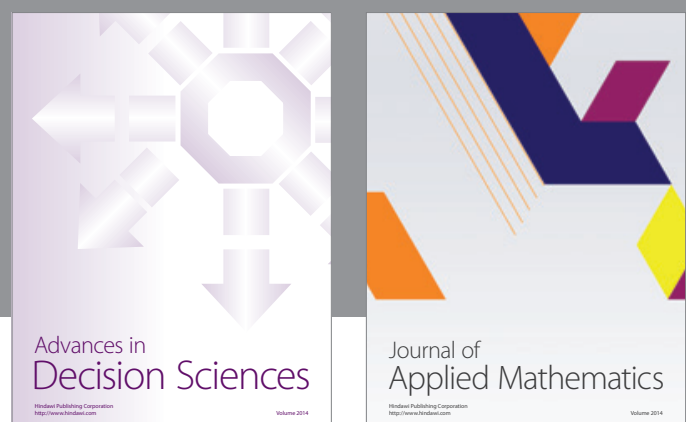

Journal of

Applied Mathematics
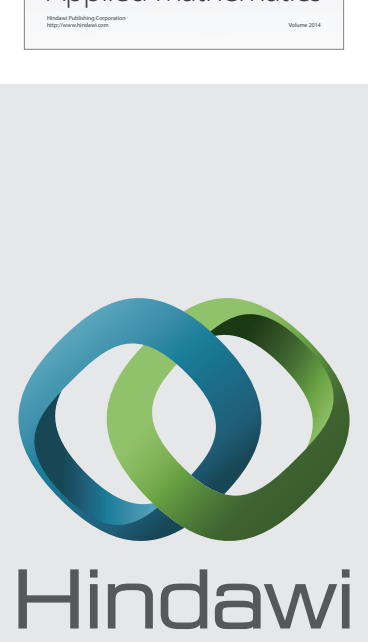

Submit your manuscripts at http://www.hindawi.com
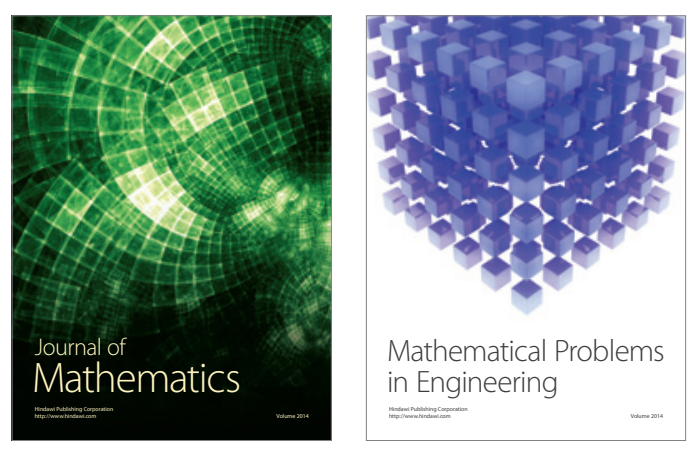

Mathematical Problems in Engineering
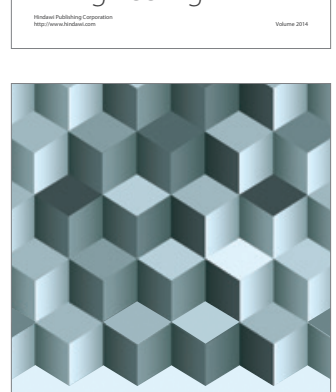

Journal of

Function Spaces
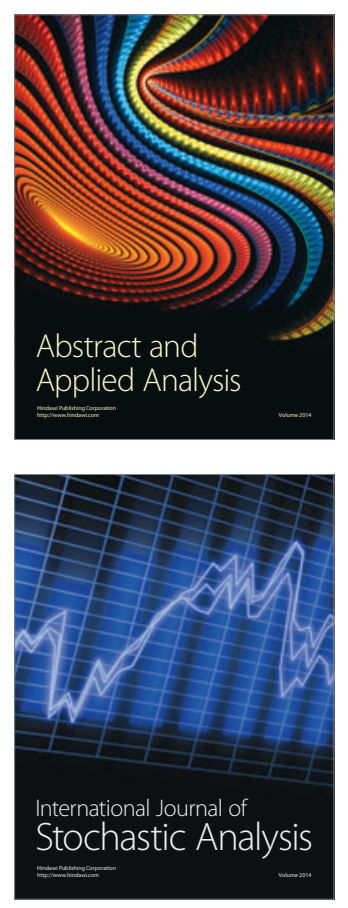

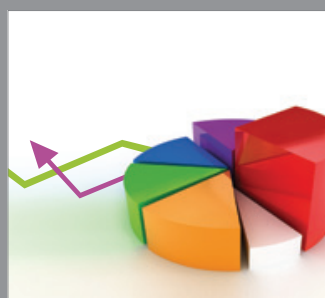

ournal of

Probability and Statistics

Promensencen
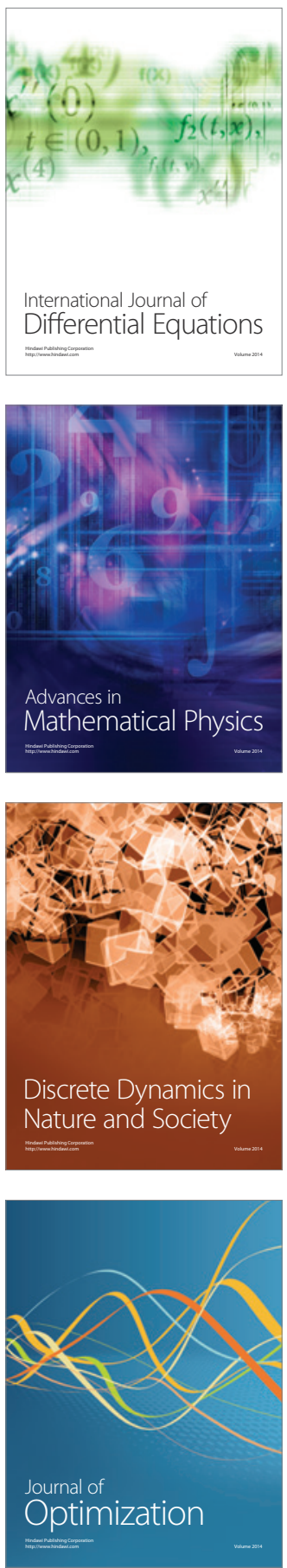